\title{
La economía política del tamaño del Estado*
}

\section{The politic economy in the size of the State}

\author{
Mireya Bermeo Álvarez**
}

Recibido: 30 de agosto de 2012

Aprobado: 7 de noviembre de 2012

\section{Resumen}

Este documento ofrece un análisis crítico de las definiciones de los determinantes políticos del tamaño del Estado, entendido como el gasto público del Gobierno nacional que se vincula a diferentes variables políticas más que económicas. Así, el gasto público corresponde con los deseos, solicitudes o demanda de los votantes por redistribución, y a su vez los gobernantes buscan maximizar su propio beneficio.

* Primer capítulo de la tesis doctoral Determinantes del tamaño del Estado. El caso colombiano. Trabajo con el cual la autora optó al título de Doctora en Ciencias Económicas. El director de este trabajo fue el doctor Fernando Tenjo Galarza y los jurados, los profesores Salomón Kalmanovitz Krauter, Mario García Molina y Manuel Muñoz Conde. Sponsor Pablo T Spiller University Of California. La defensa fue aprobada en enero de 2014. Cómo citar este artículo: Álvarez, M. (2013). La economía política del tamaño del Estado. Revista CIFE, 15 (22), pp. 87-107.

** Mireya Bermeo Álvarez. Doctora en Ciencias Económicas. Universidad Nacional de Colombia. Asesora del Ministerio de Comercio, Industria y Turismo. mbermeo@mincit.gov.co 
Esta investigación se aparta de la concepción de un gobierno benefactor o benevolente, cuyo propósito fundamental es buscar la maximización del bienestar social.

Palabras Glave: tamaño del Estado, gasto público, Gobierno, instituciones.

Clasificación JEL: H50

\section{Abstract}

This paper provides a critical survey of the definitions political determinants on the state size, understood as public expenditure of the national government, which is linked to different political variables rather than economics do. Thus, public expenditure matches with the wishes, requests or demand of the voters by redistribution, and at the same time the rulers seek to maximize their own benefit. This research did not involve the concept of a benevolent or benefactor government, whose fundamental purpose is to seek the maximization of social welfare

Keywords: Size of the state; public expenditure; government; institutions.

Classification JEL: H50 


\section{Introducción}

La literatura sobre qué determina el tamaño del Estado es amplia y variada. Su origen se remonta a la primera década del siglo XX, cuando el crecimiento del gasto público se entendía como una respuesta a las necesidades del desarrollo económico en favor de facilitar la labor productiva y proteger a los ciudadanos. Sin embargo, no es suficiente explicar el tamaño del Estado usando solo argumentos económicos, de ahí el interés de la economía política en vincular variables políticas para entender mejor las razones por las cuales el Estado crece.

Este artículo contempla la Ley de Wagner, las preferencias de los burócratas, las solicitudes o demandas de recursos por los ciudadanos, las simpatías políticas, los grupos de interés, el gobierno Leviatán y las instituciones. Dentro de estas últimas se hace mención a la constitución, a las leyes de restricciones al gasto y a la deuda pública como variable estratégica.

\section{Ley de Wagner}

La respuesta al interrogante sobre los determinantes que podrían explicar las tendencias del crecimiento del tamaño de Estado fue anticipada por Wagner en 1911. Y se refiere a dos posibles canales. Primero, argumenta que en la medida en que las economías crecen y diversifican su estructura productiva, se requieren mayores servicios por parte del Estado para proteger los derechos de propiedad, arbitrar disputas comerciales, reducir costos de transacción y resolver problemas de acción colectiva; lo que estimula la actividad productiva y el crecimiento económico, y genera un círculo virtuoso entre los niveles de ingreso y el tamaño del sector público. En segundo lugar, afirma que la elasticidad del ingreso de la demanda por bienes sociales, como la educación y la cultura, es mayor a uno, lo cual explica que cuando aumenta el producto, la demanda de estos bienes aumenta más que proporcionalmente.

Así, la elasticidad precio de la demanda y el precio determina si el Gobierno crece o se reduce. Si el precio de los bienes o servicios del Gobierno se incrementan y la cantidad demanda de bienes y servicios no disminuye en una cantidad proporcional, el gasto total aumenta. También, si el precio de los bienes y servicios del Gobierno decrece y la cantidad demanda de bienes o servicios aumenta por un monto proporcional más grande, el gasto total se incrementa.

De tal manera, con el advenimiento de la prosperidad en los países occidentales, se hicieron sostenibles las mayores demandas por redistribución, inducidas por la extensión de los derechos políticos. La prosperidad se reflejó en un mayor crecimiento económico, en mejores indicadores de salud, menores tasas de natalidad y mayor esperanza de vida. Estos cambios generaron mayores demandas por gasto social. 


\section{Preferencias de los burócratas}

Garrett y Rhine (2006: 17-27) clasificaron las teorías sobre el tamaño y crecimiento del Gobierno en dos categorías: del Estado a los ciudadanos y de los ciudadanos al Estado.

En la primera, del Estado a los ciudadanos, ${ }^{1}$ el Estado crece debido a las ineficiencias inherentes en las actividades e incentivos del sector público que enfrentan los burócratas del Gobierno.

Niskanen $(1971,2001)$, postula que los burócratas del Gobierno maximizan el tamaño del presupuesto de sus entidades de acuerdo con sus propias preferencias, dada su posición de monopolio. En esa dirección es posible un tamaño del presupuesto para las entidades públicas mayor al requerido para satisfacer las solicitudes de los ciudadanos. Y puede suceder, en primer lugar, por la dificultad de monitorear con precisión la eficiencia de producción cuando el producto disponible no es una unidad tangible. Segundo, el carácter monopólico de la mayoría de las entidades públicas les protege de presiones competitivas necesarias para mejorar la eficiencia. Tercero, como las entidades donde se asigna el presupuesto no conocen en detalle la estructura de costos de las otras entidades públicas, se presenta una oportunidad para sobre estimar los costos y recibir un presupuesto mayor. En 65 de 70 casos en los que el autor examinó el costo de prestación de servicios - en bienes idénticos- ofrecidos por los sectores públicos y privado, el costo de la prestación pública fue significativamente mayor frente a la oferta privada. Este descubrimiento apoya la teoría de la burocracia del Gobierno.

Katsimi (1998) presentó una explicación para el tamaño del sector público que se basa en la idea de "seguridad social", y planteó tres supuestos: el sector público y el sector privado producen el mismo bien; el sector público es menos eficiente frente al privado, en el sentido de que la transferencia de recursos desde el sector privado al público conduce a una caída en el consumo total; el estatus permanente del empleo público en la mayoría de países implica una menor volatilidad de la producción pública con respecto a la del sector privado. En su modelo, todos los trabajadores tienen el mismo poder de voto y pueden afectar el tamaño real del empleo público que es fijado por el Gobierno que busca maximizar la probabilidad de ser reelegido. En sus hallazgos encontró que el tamaño del sector público se establece por el Gobierno y es superior a la curva de demanda cuando el nivel de preferencias de los votantes por empleo es más grande que la pérdida por el consumo asociada con el empleo público.

Para Baumol (1967), la acumulación tecnológica en la producción o fabricación de bienes marca diferencias en el costo y en el precio real de los bienes ofrecidos por el sector privado y por el Gobierno. Muestra como el incremento en el precio de bienes o

1 State-over-citizen. 
servicios del sector público, relativo a los del sector privado, se debe a la productividad ganada en la producción. Como la mayoría de programas del Gobierno son servicios (educación, defensa nacional, etc.), no alcanza la misma eficiencia ganada en el sector privado. Así, los precios relativos de los bienes públicos se han incrementado y, con ello, el crecimiento del Estado.

Mueller (2003), siguiendo los planteamientos de Baumol (1967), presenta evidencia empírica para 25 países de la Organización de Cooperación y Desarrollo Económico (OCDE). De estos, 20 mostraron el crecimiento esperado por el efecto Baumol en los gastos del Gobierno como porcentaje del PIB para el período 1960-1995. Cuatro crecieron en alguna extensión y solo uno decreció como resultado de la reducción de gastos en defensa después de acabada la Guerra Fría.

\section{Solicitud o demanda de recursos por los ciudadanos}

La segunda categoría, según Garrett y Rhine (2006: 17-27), de los ciudadanos al Estado, ${ }^{2}$ explica el crecimiento del Estado como una respuesta a las mayores solicitudes o demandas de los ciudadanos por los programas del Gobierno y este responde al deseo de los votantes.

En este grupo se clasifican autores como Downs (1957 y 1961), quien señala como determinante fundamental del crecimiento del Estado, la extensión del voto a todos los ciudadanos, la cual puede estar asociada con una mayor demanda por bienes y servicios con altos componentes redistributivos. Esto obedece a que el votante mediano ${ }^{3}$ es más pobre y disfrutará de los beneficios de los bienes públicos en mayor medida que los costos que deberá soportar debido a los mayores impuestos. Como el Gobierno busca maximizar su soporte político en una democracia directa de mayorías, la demanda por bienes públicos del votante mediano determina su política de gasto público. En términos del acceso de la población a los derechos políticos, cuanto mayor sea el nivel de desigualdad en la distribución del ingreso en una sociedad, habrá niveles más altos de gastos redistributivos del ingreso y, por ende, un mayor tamaño del Estado.

Husted y Kenny (1997) y Lott y Kenny (1999) presentan evidencia de la relación entre la extensión del voto universal con el favorecimiento de la demanda de bienes públicos en general, y del gasto social en particular, para las ciudades y Estados de los Estados Unidos.

Meltzer y Richard (1978, 1981, 1983) presentan un modelo donde los ciudadanos seleccionan entre ocio y trabajo para maximizar su consumo. El consumo está inversamente

2 Citizen-over-state.

3 Considerado el votante decisivo en las elecciones de política pública que se adoptan por una regla de mayoría simple. 
relacionado con la tasa de impuestos y positivamente relacionado con la subvención o transferencia recibida del Gobierno; y el ingreso se relaciona positivamente con la productividad. A mayor productividad, mayor ingreso, y un ingreso más alto, incrementa el consumo y el bienestar. Estos autores muestran que los ciudadanos, bajo un mecanismo de agregación de preferencias, demandarán la combinación de tasa de impuestos y la transferencia del Gobierno que maximice su bienestar (ingreso ex post). Individuos con un nivel de productividad más bajo, y por ello un menor nivel de ingreso, demandarán una tasa de impuesto más alta y una transferencia mayor pagada del Gobierno. Hay ganadores y perdedores de la redistribución y da una función importante a la restricción presupuestal en el crecimiento del tamaño del Estado.

$\mathrm{Al}$ votar, los ciudadanos expresan sus preferencias sobre los costos y beneficios de diferentes opciones, entre ingreso ex ante e ingreso ex post, relacionadas con distintas tasas de impuestos. Así, Meltzer y Richard (1981) confirman la hipótesis según la cual el tamaño del Estado depende de la relación del ingreso medio con el ingreso del votante mediano ${ }^{-}$votante decisivo-

Mattos (2009) parte de los planteamientos de Meltzer y Richard (1981) y estima un modelo para los gastos locales y estatales de los Estados Unidos donde todas las actividades del Gobierno se basan en la distribución más que en la provisión de bienes públicos. El modelo sugiere que el crecimiento del tamaño del Gobierno federal durante la Segunda Guerra Mundial se relaciona con la expansión del sufragio universal. Los nuevos votantes tienen ingresos menores, así el votante mediano es más pobre que antes de la expansión del voto y demanda un Estado más grande. Los resultados soportan las ideas de Melzert y Richard donde una desigualdad mayor de los agentes, en términos del ingreso, determina un tamaño del Estado más grande.

Shelton (2007) utilizó datos estadísticos financieros de los Gobiernos, los obtuvo del Fondo Monetario Internacional, con series de tiempo y corte transversal para una muestra de más de 100 países en el período 1970-2000, y encontró que el nivel de desigualdad y el acceso político, afectan el tamaño del gasto público.

Acemoglu y Robinson (2006) realizaron una extensión del modelo de Melzert y Richard (1981). Pasan de democracias directas a representativas, donde el elegido implementa la política redistributiva que escoge el votante mediano, y si estos son más pobres, aumenta el tamaño del Estado. Cuando cambian de políticas de interés general a particular, el conflicto de intereses entre ricos y pobres se genera con respecto al bien público deseado y a la tasa de impuesto. Como los pobres son mayoría, la política preferida induce a un Estado más grande. Con las trasferencias focalizadas, el bien público se dirige al segmento de la población donde se concentra la mayoría de votantes, es decir, el votante mediano. En este escenario es importante saber a quién se dirigen las transferencias; la 
tasa de impuestos de equilibrio es mayor porque, intuitivamente, como la distribución va a un solo grupo, el beneficio neto es mayor, con lo cual el tamaño del Estado crece.

Con un modelo intertemporal, Alesina y Giuliano (2009), evalúan los factores que determinan las preferencias por redistribución en los Estados Unidos. Los hallazgos encontrados en relación con el ingreso siguen los planteamientos de Meltzer y Richard (1981). Cuando agregan movilidad social y la expectativa futura es de un ingreso mayor, los votantes prefieren menos redistribución y un Estado más pequeño. En su análisis con la raza encontraron que los blancos, quienes representan la mayoría de la población, son menos favorables a la redistribución, frente a la minoría negra que prefiere una distribución mayor.

\section{Simpatías políticas}

Kristov, Linder y McClelland (1992) presentaron un modelo que examina las simpatías políticas basadas en la afinidad sobre la distribución del ingreso. Estudiaron el período precedente y durante la Gran Depresión. Explican que cuando la economía estuvo en expansión, los americanos votaron en contra de aumentar los impuestos para redistribuir a los pobres. Pero después del cambio de la dirección de la economía en los años treinta, los impuestos a los ingresos aumentaron y el número de programas que redistribuye el ingreso y la riqueza también se incrementaron.

Desdoigts y Moizeau (2005) sugieren un modelo para explicar por qué una mayor desigualdad social puede estar vinculada con un menor número de votantes que demandan políticas más redistributivas, en una democracia donde el nivel de redistribución se decide por votación de mayorías. Construyen sus argumentos sobre la base de la importancia que las aspiraciones de los miembros de una comunidad tienen, como fuente de inspiración, para cada individuo cuando revelan sus preferencias sobre el alcance de las políticas redistributivas, alejándose del teorema del votante mediano. Ofrecen una explicación de por qué habría menos presión por redistribución en economías más desiguales y segmentadas como, por ejemplo, en los Estados Unidos, en comparación con economías caracterizadas por una población más homogénea y con movilidad ascendente en el ingreso; es el caso de los países escandinavos donde las solicitudes por redistribución son más grandes.

Lijphart (1997) y Bénabou (1996 y 2000) presentan una paradoja en la política pública por cuanto, según sus hallazgos, el Estado redistribuye menos, en presencia de una fuerte desigualdad en la distribución del ingreso en contextos de sistemas electorales democráticos. Muestran que los ciudadanos de mayores ingresos son los que más ejercen su derecho al voto y los que tienen más alcance a formas alternativas de participación política (lobby y contribuciones al financiamiento de las campañas, por ejemplo). Dada la diferencia entre la participación de jure y de facto, y en vista de que los ciudadanos de altos ingresos están 
mejor representados en el proceso político, se presenta una menor demanda por redistribución. Esta relación negativa entre desigualdad y gastos de redistribución se documenta en economías desarrolladas y en vía de desarrollo (Lindert, 2004).

\section{Grupos de interés}

Los grupos de interés también pueden incrementar el tamaño del Estado. Cuando los miembros de una organización hacen presión política son más efectivos que los ciudadanos individualmente, no obstante los problemas del gorrón ${ }^{4}$ (Olson, 1965, Moe, 1980).

Un grupo de interés puede ser pensado como una colectividad de votantes organizados que tienen las mismas preferencias por una política específica. Por medio del lobby, un grupo de interés puede obtener una política deseada que tenga beneficios directos para ellos, y hacer que los costos de la política se esparzan entre millones de contribuyentes. La falta de conexión entre los costos y los beneficios suscita un gasto público ineficiente, así, el costo social de la política es mayor frente al beneficio social.

Es probable que nadie quiera renunciar a los beneficios derivados de un gasto mayor cuando no se responsabilice del costo de pagar impuestos más altos. Como el presupuesto se financia de manera centralizada, en el entendido de que los recursos fiscales se obtienen de parte de la sociedad como un todo y los grupos que ganan de la redistribución buscan un nivel de gasto mayor, ello conduce a una situación conocida en la literatura de la Económica Política como la "Tragedia de los Comunes", ${ }^{5}$ donde cada grupo pretende conseguir la mayor cantidad de recursos del fondo común del presupuesto, eludiendo el compromiso del pago de impuestos. Este es quizá, uno de los problemas políticos más importantes que enfrenta el presupuesto público.

Chamon y Kaplan (2013) presentaron un modelo sobre la forma de financiamiento de las campañas para las elecciones a la Cámara de Estados Unidos en el período 19841990, considerando aportes de grupos de interés. Encuentran que estos, a veces, pueden obtener apoyo sin gastar dinero, e incluso, el dinero que gastan solo refleja la superficie de su influencia. Sugieren que la conexión entre el dinero gastado y el efecto del dinero en la política no es simple. Por ello, centrar el trabajo empírico en contribuciones de grupos de interés a las campañas puede subestimar su influencia.

Weingast, Shepsle y Johnson (1981) analizaron políticas redistributivas que concentran beneficios en un distrito especial mediante un proyecto específico, financiado por los contribuyentes dispersos en todo el país. Señalan como cada región de la circunscripción

4 La figura del gorrón, polizón o "free-rider" se entiende como aquel que disfruta de los beneficios de una acción colectiva sin participar en ella.

5 "Estas teorías buscan explicar el sesgo político hacia acumular deuda como la consecuencia de que los recursos que se extraen para satisfacer las demandas de las regiones o grupos representados políticamente hacen parte de una 'piscina común”". Echeverry, Bonilla y Moya (2006: 17). 
no toma para sí, el costo de los proyectos de otras localidades. En consecuencia, el beneficio neto de un proyecto está sobreestimado. Sin embargo, los políticos o gobernantes de dichas localidades tienen gran interés en el mismo por el respaldo que puedan recibir de su región.

Becker (1983) planteó una teoría de la política pública como el resultado de la competencia entre los grupos de presión y los asume como un bien público. ${ }^{6}$ Encontró que la eficiencia en la producción de la presión está parcialmente determinada por los costos para controlarla. Un control más grande sobre los gorrones, incrementa la cantidad de presión. Con niveles más altos de presión, un grupo de presión especial puede adquirir reducción de impuestos o mayores subsidios. Por lo tanto, la interacción entre los grupos de presión en competencia aumenta el poder del lobby, y con ello, el gasto del grupo de interés.

Aunque la teoría de los grupos de interés puede proveer, al menos en parte, una explicación razonable para el tamaño del Estado, aún no está libre de problemas como el de la causalidad. Concretamente, como lo plantean Mueller y Murrell (1985, 1986), ¿la actividad de los grupos de interés causan los gastos del Gobierno, o los cambios en el nivel de los gastos del Gobierno influencian la actividad de los grupos de interés?

\section{Gobierno Leviatán}

Teorías como la de Joulfaian y Marlow (2001), asimilan los modelos de la elección pública y el comportamiento monopólico del Gobierno a la conducta del Leviatán. Estos, son tomados de la literatura económica de la organización industrial. Siguen el argumento del monopolio privado en la producción donde los precios y los beneficios son relativamente altos.

La hipótesis del Gobierno Leviatán sugerida por Brennan y Buchanan (1977, 1980), es motivada por el deseo de entender correctamente el comportamiento del Gobierno y para recomendar medidas eficaces en el control de su conducta. Los ciudadanos, se supone, pierden todo el control de su Gobierno y la competencia política es vista como una restricción inefectiva sobre el crecimiento del mismo. El Gobierno Leviatán es entendido como una entidad pública que busca maximizar su propio ingreso, y no como un proveedor benevolente de mercancías, un reductor de las externalidades, y un redistribuidor de renta.

Para Brennan y Buchanan (1977, 1980), solo las restricciones constitucionales sobre la autoridad del Gobierno, la descentralización de los impuestos, y el poder del gasto de los Gobiernos y los asuntos de deuda, pueden limitar a un Gobierno Leviatán. Presentan evidencia empírica donde soportan que la competencia entre las distintas unidades

6 Un bien público es no rival - el consumo de una persona no impide el consumo de otros- y no excluyente, porque no existen mecanismos de precios para negar el consumo. 
fiscales de los Gobiernos locales es un medio eficaz para controlar el tamaño central del Leviatán. La concentración de los impuestos en manos del Gobierno central expande su rango, sobre el cual puede aplicar su poder monopólico. Cuanto mayor es el rango de actividad económica gravada por el Gobierno central, es probable que el tamaño total del sector público sea más grande. El resultado de la descentralización se fundamenta en que una intervención en la economía debería ser más pequeña, ceteris paribus, dado el mayor grado de descentralización en los impuestos y en los gastos. La autonomía fiscal conduce a una competencia territorial que limita el tamaño del sector público y aumenta la eficiencia. El corolario es una demanda de descentralización que tenga en cuenta las preferencias locales, forzosamente heterogéneas (Pérez, 2008).

Joulfaian y Marlow (2001), probaron la hipótesis de la descentralización al examinar un conjunto de datos que considera todas las unidades del Gobierno en un estudio para los estados de Estados Unidos en los años 1981 y 1984, y ofrecen evidencia empírica que soporta la hipótesis de Brennan y Buchanan $(1977,1980)$ en el sentido de que la descentralización fiscal tiene un efecto inverso asociado con el tamaño total del Estado.

Grossman (1992) examinó el impacto de la descentralización fiscal, la colusión intergubernamental y la dependencia fiscal en la determinación del tamaño del sector público en Australia, entre los años 1950 y 1984. Definió el sector público como un porcentaje del total de los gastos del Gobierno en relación con el PIB. En dicho período, este creció del 28,2 \% al 41,5\%, donde los gastos de los Gobiernos, estatal y local, como porcentaje de los gastos totales del Gobierno, pasaron del 42,7 \% en 1950, al 47,0 \% en 1984. La evidencia no apoyó la hipótesis de la descentralización planteada por Brennan y Buchanan (1977, 1980); aunque el autor encontró una correlación positiva entre la descentralización y el tamaño del sector público, no fue estadísticamente significativa. Presenta tres posibles explicaciones para esos resultados: el número pequeño de Gobiernos sub-nacionales; la insignificancia económica de los Gobiernos locales (representan aproximadamente el $5 \%$ del total de los gastos del sector público) y la relativa inmovilidad de los ciudadanos.

A diferencia de la descentralización, halló evidencia de que la colusión contribuye al tamaño del Estado. También, la dependencia fiscal, definida como la proporción de las subvenciones de la Commonwealth a los Gobiernos estatales y locales, sobre el total de las finanzas estatales y locales, demuestra una correlación positiva y significante con el tamaño del sector público.

Grossman (1992) sugiere medir la descentralización como la independencia fiscal de los Gobiernos sub-nacionales del Gobierno otorgante, o sea, los gastos del Gobierno de nivel sub-nacional financiados con rentas propias como porcentaje de los gastos totales del Gobierno. En este caso, estos declinaron del 28,7 \% al 24,0 \%, entre 1950 y 1984 , en Australia. 
También Oates $(1972,1989)$ reportó evidencia empírica contraria a los hallazgos de Brennan y Buchanan (1977, 1980). Oates vincula el tamaño del sector público con la atribución de autoridad a los niveles sub-nacionales para el ejercicio de las finanzas. Analiza el comportamiento de la descentralización de los Gobiernos estatales y locales de 43 países, y sus pruebas no apoyan la hipótesis del efecto inverso asociado con la descentralización para controlar el crecimiento del Estado. En el mismo sentido, Nelson (1986), en sus test empíricos, no encontró soporte para apoyar la hipótesis de la descentralización.

Muchos estudios empíricos examinan, con resultados diferentes, el efecto de la competencia intergubernamental sobre el tamaño agregado del Estado. Hallazgos que dependen del periodo de tiempo que se analiza y, sobre todo, de la forma como se mida la descentralización.

Pérez (2008) examinó el impacto de la descentralización en España sobre el tamaño de los Gobiernos regionales, donde el gasto gestionado por la administración central descendió en términos relativos - de un 76,6 \% del total en 1985 a un 53 \% en 2004-en beneficio de las Administraciones Territoriales, cuyo peso aumentó hasta en un 33,4 \%, la autonómica (Gobierno regional), y un 12,6 \% la local. En sus resultados, rechaza la hipótesis de contención del Leviatán, pues la autonomía fiscal parece aumentar, en lugar de reducir, como lo sugiere una parte de la literatura, el tamaño del sector público. Muestra que los Gobiernos sub-nacionales crecen más cuando obtienen una parte importante de los recursos financieros a través de transferencias intergubernamentales, pues la descentralización de gastos sin la adecuada descentralización de ingresos, diluye la competencia fiscal y la responsabilidad moral, así, el desequilibrio presupuestario aumenta la incongruencia entre los que se benefician de los programas y quienes los pagan.

Micklethwait (2011) señaló que países como India y China se preguntan qué tipo de Estado necesitan para satisfacer las demandas de sus ciudadanos con el fin de mejorar las escuelas, los servicios de salud y la infraestructura ¿Cómo puede hacerse un Gobierno más eficiente? ¿Qué debe hacer y qué no hacer? ¿A quién debería responder? El argumento central es que el Leviatán puede ser más eficiente. Con demasiada frecuencia una institución, al menos en una democracia, que se supone sirve a los ciudadanos, se ha convertido en su jefe. Los estados han tendido a crecer impulsados principalmente por los pagos de transferencias y subvenciones supuestamente destinados a los pobres, pero a menudo el mayor beneficio es para la clase media y alta. En general, no se trata de corrupción en todos los políticos, sino que ellos no entregan los servicios que la gente quiere.

Drazen (2000, pp. 23-24) señala problemas de agencia en la toma de decisiones en temas como las transferencias. El principal (Gobierno central), delega en el agente (Gobiernos sub-nacionales), con diferencias en las preferencias, la asignación del gasto. En general, el agente no reporta información crucial al principal, a menos que reciba 
un incentivo para hacerlo. En la descentralización, donde las decisiones de recaudo son separadas de las decisiones del gasto, el agente tiene incentivos para aumentar el tamaño del Estado.

\section{Las instituciones}

\subsection{La constitución}

Persson y Tabellini $(2004,2005)$ evaluaron algunas características de las constituciones y su papel en la determinación del tamaño del Estado. Tomaron dos elementos: las formas del Gobierno - presidenciales o parlamentarias-y las reglas electorales -de mayoría o proporcionales-, y encontraron, según la evidencia empírica de su modelo, regímenes presidenciales con Gobiernos pequeños frente a las democracias parlamentarias con Gobiernos grandes, y que las elecciones de mayorías se asocian con Gobiernos y programas de bienestar pequeños en comparación con el sistema proporcional electoral.

En participación democrática, Persson y Tabellini (2002) mostraron que en las democracias más representativas, la consciencia de los políticos sobre su período de gobierno por un término finito y su posible remplazo o los cambios de su partido en el poder, son características con numerosas implicaciones sobre el gasto público.

Eslava y Nupia (2010) evaluaron la hipótesis según la cual la fragmentación de partidos políticos debe afectar el gasto del Gobierno. Sus resultados para democracias presidenciales plantearon que es el nivel de fragmentación en la coalición de Gobierno, más que el nivel de fragmentación de los partidos políticos en la legislatura en su conjunto, lo que afecta positivamente el tamaño del gasto.

Bermeo (2014) encontró un vínculo entre el crecimiento del tamaño del Estado colombiano con la Constitución Política de 1991, la elección popular de alcaldes en 1986, la relación inversa de la tasa de ingreso per cápita y la deuda, en la evidencia empírica para Colombia. De hecho, con la Constitución de 1991 se orientó conceptualmente el gasto hacia la descentralización, materializada a través del Sistema General de Participación y la elección popular de alcaldes y de gobernadores. Además, en esta se expresan las nuevas demandas o solicitudes por redistribución, ${ }^{7}$ donde se encuentra en los principios y derechos: la igualdad y la justicia, las pensiones, la seguridad social, la vivienda digna y el pago oportuno de los sueldos del sector público, entre otros (artículos 42 a 56). Adicionalmente, dio prioridad al gasto público social ${ }^{8}$ sobre cualquier otra asignación,

7 Según los cinco últimos marcos fiscales de mediano plazo (2003-2008), sin incluir el servicio de la deuda, el mayor gasto se ha concentrado en protección social, defensa y seguridad y educación.

8 El presupuesto de inversión del gasto social no se puede disminuir porcentualmente en relación con el año anterior respecto del gasto total de la correspondiente ley de apropiaciones. Artículo 366 de la Constitución Política de 1991, modificado por el Acto Legislativo. 
fijó la garantía del cumplimiento del Estado Social de Derecho con instituciones como la Corte Constitucional; la acción de tutela; la eliminación de la obligación de presentar presupuestos balanceados sin establecer límites cuantitativos al crecimiento del tamaño del Estado y la restricción al crecimiento de la deuda como la capacidad de pago de la Nación y de los entes territoriales (artículo 364).

\subsection{Leyes de restricciones al gasto}

McCubbins, Kousser y Moule (2005) buscan responder si en los votantes o los legisladores, a través de leyes donde se establecen limitaciones del gasto tienen efecto sobre la reducción del tamaño del gasto público o son solo letra muerta. Estos autores se basan en la literatura sobre agente - principal, y demuestran cómo las limitaciones fiscales y de gastos pueden ser eludidas por inspiración de los funcionarios electos. Si los gobernantes responden a la demanda de sus electores, evadiendo los límites, al camuflar gastos en los engorrosos documentos presupuestales, crece la dificultad de hacerles seguimiento. ${ }^{9}$ De esta manera, las limitaciones fiscales y de gastos, por sí mismas, no son mecanismos eficaces de control del tamaño del Estado.

McCubbins (1995) analizó por qué las proposiciones 4, 13, 98 y 99, mediante las cuales los votantes buscan comprometer la responsabilidad del Gobierno de California en el gasto, lo han limitado al punto que le es difícil responder a las demandas de los ciudadanos en materia económica, social y política. Como los controles no son claros y los compromisos son compartidos con las Cámaras, no es fácil establecer la responsabilidad de los políticos. Así, cuando los votantes ponen una restricción sobre los gastos en un área, los legisladores se mueven rápidamente a otra, y el único modo de escapar de este círculo es atender las necesidades legales de incentivos para gastar tanto como el dinero de quienes pagan impuestos lo permita.

Bails y Tieslau (2000) proveen una evaluación empírica para 49 de los 50 estados federados de los Estados Unidos entre 1969 y 1994. Consideran el efecto de las limitaciones fiscales en el gasto real per cápita estatal y local en Estados Unidos. Encontraron gastos inferiores en los estados donde se tienen límites fiscales y de gastos, y exigencia de balance presupuestal frente a donde estas no existen. Demuestran como un Estado con mecanismos de disciplina fiscal, enfrenta gastos per cápita reales aproximadamente 14 veces más bajos en relación con aquellos donde no se tiene.

Por su parte, Elder (1992) analizó la efectividad de las leyes aprobadas para limitar el crecimiento de las rentas o gastos del Gobierno en los Estados de Estados Unidos, en particular, el efecto de esas leyes sobre la carga de impuestos, durante el periodo 19501985. Resaltó como las leyes de limitación de rentas y gastos pueden ser instrumentos

9 La evidencia empírica la aportan para los estados de los Estados Unidos, comparando los patrones fiscales de cada uno, antes y después de la adopción de las leyes donde se establecen las limitaciones fiscales y de gastos. 
efectivos para contener la carga de impuestos. El éxito, según el autor, depende no solo de las provisiones de una ley, sino también del conjunto de mecanismos por el cual la disciplina fiscal es impuesta en un Estado.

Según Eslava (2006), los votantes tienden a ser conservadores fiscales, pero enfrentan dificultades en el monitoreo del gasto del Gobierno y en la elección de impuestos, por ello la disciplina fiscal debe ser aumentada por límites numéricos al déficit.

Cuando opera una restricción al gasto, la lucha por los recursos lleva, probablemente, a una reasignación, pero cuando esta se elimina, incentiva un aumento en la deuda y con ello, el tamaño del Estado.

\subsection{La deuda como variable estratégica}

Políticamente, la deuda, como lo señalaron Alesina y Tabellini $(1990),{ }^{10}$ permite al Gobierno que está en el poder gastar más, y además, trasladar su pago al gobernante futuro. Los autores presentan un modelo donde el gobernante de turno aumenta estratégicamente el gasto y genera déficit para atar las manos de sus sucesores a través de la deuda. Si el gobernante de turno se enfrenta con un alto riesgo de ser remplazado por alguien de un partido diferente, es posible que aumente el gasto en bienes de su preferencia toda vez que el costo del déficit, probablemente, será pagado por su sucesor.

La naturaleza política de la deuda no sólo genera redistribución entre las generaciones presentes. Alesina y Perotti (1995, pp. 13-16), en su obra Economía Política de los Déficits Presupuestales, señalaron cómo la naturaleza intertemporal de las decisiones fiscales crea vínculos entre las generaciones. Por ello, la deuda pública puede generar redistribución tanto intrageneracional como intergeneracional, si las que están vivas hoy dejan la carga de la deuda a las futuras.

En principio, una generación egoísta podría votar por políticas que cambien la carga de los impuestos al futuro. Como resultado se estaría pidiendo prestado, indirectamente, de las generaciones por nacer.

La literatura de la Economía Política también da explicaciones de por qué el pago de la deuda se pospone. Alesina y Drazen (1991) analizaron la acumulación de la deuda como el resultado de una guerra de espera, de aguante o de desgaste, situación que demora hacer los ajustes fiscales; a causa de la polarización ideológica. El modelo supone dos grupos y ninguno sabe cuánto puede resistir el otro, por lo tanto, el que tenga mayor capacidad de espera o aguante (ganador), logra trasladar el costo mayor de la carga fiscal de la estabilización al perdedor.

10 En su modelo tienen en consideración la relación entre la competencia de partidos, la polarización de preferencias y la incertidumbre electoral. Los factores políticos institucionales resultan cruciales para entender el comportamiento de la deuda y, por ende, su efecto en el crecimiento del tamaño del Estado. 
Spolaore (citado en Alesina y Perotti, 1995) aplicó un modelo de guerra de espera a Gobiernos de coalición y demuestra que estos demoran los ajustes fiscales. Este resultado surge porque diferentes partidos representan el interés de electores distintos. Menor cohesión implica, mayor dificultad para alcanzar un acuerdo sobre una distribución equitativa de los costos del ajuste fiscal y, por lo tanto, una gran demora en detener el crecimiento de la deuda. Además, las instituciones políticas y las leyes electorales que conducen a la formación de coaliciones de Gobierno, podrían estar asociadas con déficits mayores frente a Gobiernos de un solo partido. De esta manera, se podría sugerir una relación entre la forma del sistema electoral y el nivel de deuda.

Asimismo, Alesina y Perroti (1995), advirtieron como la naturaleza intertemporal de las decisiones de gastos e impuestos crean vínculos a través de las generaciones y pueden dejar legados negativos a quienes aún no han nacido; es el caso de la deuda, que se refleja en el crecimiento del tamaño del Estado.

Como lo señalaron Fergusson y Querubin (2010), existen tres elementos fundamentales planteados en la aplicación de las normas: las restricciones a los gobernantes deben estar normadas; las políticas no se aplican en el vacío y se desarrollan dentro de un contexto específico; y la formación de rentas y la asignación del gasto es un proceso similar a favor por favor se paga.

\section{Conclusiones}

Las definiciones sobre el tamaño del Estado, que esbozan esta investigación, invitan a pensar en el gasto público como una decisión política más que económica. Lo cual vincula el tamaño del Estado con las políticas redistributivas de cada sociedad en los diferentes momentos del tiempo.

El interés por explicar los determinantes del tamaño del Estado se remonta a los tiempos de Wagner (1911). Para este autor, un mayor crecimiento de la economía conduce a un Estado más grande. De igual manera, como la elasticidad del ingreso de la demanda por bienes sociales es mayor a uno, cuando aumenta el producto, la demanda de los bienes ofrecidos por el Estado crece más que proporcionalmente.

Para algunos investigadores de la economía política, el Estado crece por las ineficiencias inherentes a las actividades e incentivos ofrecidos por el sector público. Mientras para otros, el crecimiento del Estado obedece a las mayores solicitudes o demandas de los ciudadanos por programas del Gobierno, y este responde al deseo de los votantes. De ahí la importancia de las instituciones en el gasto público. 


\section{Referencias}

Acemoglu, D. Robinson, J. (2006). Economic origins of Dictatorship and Democracy. Cambridge University Press. http://www.international.ucla.edu/media/files/PERG.acemoglu. pdf

Alesina, A. Perotti, R. (1995). The Political Economy of Budget Deficits. IMF Staff Papers. International Monetary Fund Palgrave Macmillan Fournals, 42(1), 1-31. Recuperado de: www.jstor.org/stable/3867338

Alesina, A. Drazen, A. (1991). Why Are Stabilizations Delayed? American Economic Review, 81(5), 1170-1188. Recuperado de: http://www.jstor.org/stable/2006912

Alesina, A., \& Giuliano, P (2009). Preferences For Redistribution. Working paper 14825. Recuperado de: http://www.nber.org/papers/w14825 .

Alesina, A. Tabellini, G. (1990). A positive theory of fiscal deficits and government debt. Review of Economic Studies. 57(3), 403-414. Recuperado de: http://nrs.harvard.edu/ urn-3:HUL.InstRepos:3612769

Bails, D. Tieslau, M. (2000). The Impact of Fiscal Constitution on State and Local Expenditures. Cato Fournal, 20(2), 255-77. Cato Institute. Recuperado de: www.cato. org/sites/cato.org/files/.../cj20n2-7.pdf. http://object.cato.org/sites/cato.org /files/serials/files/cato-journal/2000/11/cj20n2-7.pdf

Baumol, W. (1967). The Macroeconomics of Unbalanced Grhowth: The Anatomy of Urban Crisis. American Economic Review, 57(3), 415-26. American Economic Association. Recuperado de: http://www.jstor.org/stable/1812111

Becker, G. (1983). A Theory of Competition among Pressure Groups for Political Influence. Quarterly Fournal of Economics, 98(3), 371- 400. Harvard University. Recuperado de: http://www.jstor.org/about/terms.html. http://www.jstor.org/stable /1886017

Bénabou, R. (1996). Inequality and growth. Ben Bernanke y J. Rotemberg (Eds.), NBER Macroeconomics annual, pp. 11-92. Boston: MIT Press. Recuperado de: http://www. nber.org/chapters/c11027

Bénabou, R. (2000). Unequal societies: Income distribution and the social contract. The American Economic Review, 90(1), 96-129. American Economic Association. Recuperado de: www.princeton.edu/ rbenabou/papers/AER.pdf http://www.jstor.org/discov er $/ 10.2307 / 117283$ ?uid $=3737808 \&$ uid $=2134 \&$ uid $=2 \&$ uid $=70 \&$ uid $=4 \&$ sid $=2110$ $4568152713 \mathrm{~g} /$ discover $/ 10.2307 / 117283$ ? uid $=3737808 \&$ uid $=2134 \&$ uid $=2 \& u i d=7$ 0\&uid=4\&sid $=21104568152713$ 
Bermeo, M (2014). Determinantes del tamaño del Estado. El caso Colombiano. Tesis presentada como requisito para optar al título de Doctora en Ciencia Económicas. Universidad Nacional de Colombia.

Brennan, G. Buchanan, J. (1977). Towards a Tax Constitution for Leviathan. Fournal of Public Economics, 8(3), 255-73. Recuperado de: http://dx.doi.org/ 10.1016/0047-2727(77)90001-9

Brennan, G. Buchanan, J. (1980). The Power to Tax: Analytical Foundations of a Fiscal Constitution. Cambridge: Cambridge University Press. Vol. 9. Recuperado de: www. econlib.org/library/Buchanan/buchCv9.html. http://www.econlib.org/library/Buchanan/buchCv9c0.html\#Preface

Chamon, M. Kaplan, E. (2013). The Iceberg Theory of Contributions: Political Threats and Interest Group Behavior. American Economic fournal: Economic policy. 5(1), 1-31. Recuperado de: http://econweb.umd.edu/ kaplan/jmp_final.pdf

Desdoigts, A. Moizeau, F. (2005). Community Membership Aspirations: The Link Between Inequality and Redistribution Revisited. International Economic Review. 46(3), 973 -1007. Recuperado de: http://onlinelibrary.wiley.com/doi/10.1111/ iere.2005.46.issue-3/issuetoc

Downs, A. (1957). An Economic Theory of Political Action in a Democracy. Journal of Political Economy, 65(2), 135-150. Recuperado de: http://www.jstor.org/ stable/1827369

Downs, A. (1961). Problems of Majority Voting: In defense of Majority Voting. Journal of Political Economy, 69(2), 192 -99. Recuperado de: http://www.jstor.org/ stable/1828726

Drazen, A. (2000). Political Economy in Macroeconomics. New Jersey: Princeton University Press. Recuperado de: http://press.princeton.edu/titles/6819.html

Echeverry, J. C., Bonilla, J. A. y Moya, A (2006). Rigideces institucionales y flexibilidad presupuestaria: los casos de Argentina, Colombia, México y Perú. Bogotá: CEDE. ISSN 16577191. Recuperado de: http://economia.uniandes.edu.co/publicaciones/d2006-33. pdf

Elder, H. W. (1992). Exploring The Tax Revolt: An Analysis of The Effectiveness of State Tax and Expenditure Limitation Laws. Public Finance Review, (20), 47-63. Recuperado de: http://pfr.sagepub.com/content/20/1/47

Eslava, M. (2006). The Political Economy of Fiscal Policy: Survey. Inter-American Development Bank (BID). (Research Department Working Paper No. 583). Bogotá: Universidad de 
los Andes. Recuperado de: http://www.google.com.co/url?sa=t\&rct=j\&q=\&esrc=s $\&$ frm $=1 \&$ source $=$ web $\& c d=1 \&$ cad $=$ rja\&uact $=8 \&$ ved $=0 \mathrm{CCgQFjAA} \& u r l=\mathrm{http} \% 3 \mathrm{~A}$ $\% 2 \mathrm{~F} \% 2 \mathrm{Fwww}$.iadb.org $\% 2$ Fres $\% 2$ Fpublications $\% 2$ Fpubfiles $\% 2$ FpubWP-583.pdf\& ei=MqniU4DqG6rNsQSpxoHYGA\&usg=AFQjGNFgTI-eTiivRlxDdWTSwUUdRYwmQA http://www.iadb.org/res/publications/pubfiles/pubWP-583.pdf

Eslava, M. Nupia, O. (2010). Political Fragmentation and Government Spending: Bringing Ideological Polarization Into The Picture. Documentos CEDE ISSN 16575334. Bogotá: Universidad de los Andes. Recuperado de http:/ /www.google.com.co/ url? $\mathrm{sa}=\mathrm{t} \& \mathrm{rct}=\mathrm{j} \& \mathrm{q}=\& \mathrm{esrc}=\mathrm{s} \& \mathrm{frm}=1 \&$ source $={ }_{\mathrm{web}} \& \mathrm{~cd}=2 \& \mathrm{cad}=\mathrm{rja} \&$ uact $=8 \& \mathrm{ved}=$ 0CCIQFjAB\&url=http $\% 3 \mathrm{~A} \% 2 \mathrm{~F} \% 2 \mathrm{Feconomia}$.uniandes.edu.co $\% 2 \mathrm{Fpublicacione}$ s\%2Fdcede2010-03.pdf\&ei=9aniU9y2MvbLsASpyoKQAw\&usg=AFQjCNGGgrr eRQN7N1znB_aVWMoBwI4-7Q http://ideas.repec.org/p/col/000089/006713. html http://www.urosario.edu.co/urosario_files/cb/cbe552b1-00db-40c7-b09e82fb6ee3f194.pdf

Fergusson, L. Querubin, P. (2010). Política, economíay política económica. Una introducción a la nueva economía politica. Bogotá: Universidad de los Andes. Recuperado de: economia.uniandes. edu.co/content/.../file/Lecture_Notes_w_Figures.pdf. http://www.google.com.co/ url? sa $=t \& r c t=j \& q=\& e s r c=s \& f r m=1 \&$ source $=$ web\&cd $=1 \& v e d=0$ CBoQFjAA\&url $=h$ ttp $\% 3 \mathrm{~A} \% 2 \mathrm{~F} \% 2 \mathrm{Feconomia}$.uniandes.edu.co $\% 2 \mathrm{~F}$ content $\% 2 \mathrm{Fdownload} \% 2 \mathrm{~F} 39922 \%$ 2F350057\%2Ffile\%2FLecture_Notes_w_Figures.pdf\&ei=-gDkU87nJZPjsASsz4KAB w\&usg=AFQjCNFney_bcq-cqSZNvlq-342kbyPAdw\&bvm=bv.72676100,d.cWc

Garrett, T. Rhine, R. (2006). On the Size and Growth of Government. Federal Reserve Bank of St. Louis Review, 88(1), 13-30. Recuperado de: http://research.stlouisfed.org/ publications/review/06/01/GarrettRhine.pdf

Grossman, P. (1992). Fiscal Decentralization and Public Sector Size in Australia. The Economic Record, 68(3), 240-246. Recuperado de: http://onlinelibrary.wiley.com/doi/10.1111/j.1475-4932.1992.tb01770.x/. http://www.blackwell... bs.asp?ref=00130249. http://EconPapers.repec.org/RePEc:bla:ecorec:v:68:y:1992 :i:202:p:240-46

Husted, T. Kenny, L. (1997). The Effect of the Expansion of Voting Franchise on the Size of the Government. Fournal of Political Economy, 105(1), 55-82. Recuperado de: http://papers.ssrn.com/sol3/papers.cfm?abstract_id=4628

Joulfaian, D. Marlow, M. (1990). Government and Decentralization: Evidence from Disaggregated Data, Southern Economic Journal, 56, (4), 1094-1102. Southern Economic Association. Recuperado de: http://www.jstor.org/stable/1059894 
Katsimi, M. (1998). Explaining the Size of the Public Sector. Public Choice, (96), 117-144. Springer. Recuperado de: http://www.jstor.org/stable/30024381

Kristov, L., Linder, P. McGlelland, R. (1992). Pressure Groups and Redistribution. Elsevier Fournal of Public Economics, 48(2), 135 -63. Recuperado de: http:/ /www.sciencedirect.com/science/article/B6V76-458X2Y31F/2/6f9bc9284c7749f7ba874978 b81aa8fa http://www.sciencedirect.com/science/article/pii/004727279290024A

Lijphart, A. (1997). Unequal Participation: Democracy's Unresolved Dilemma. The American Political Science Review, 91(1), 1 -14. American Political Science Association Recuperadodehttp://www.jstor.org/stable/2952255

Lindert, P. (2004). Social Spending and Economic Growth Since the Eighteenth Century Growing Public. Cambridge: Cambridge University Press. http://www.amazon.es/Growing -Public-Paperback-Spending-Eighteenth/dp/0521529166

Lott, J. Kenny, L. (1999). Did Women's Suffrage Change the Size and Scope of Government? Journal of Political Economy, 107(6), 1163-1198. Recuperado de: http:/ / www.people.fas.harvard.edu/ iversen/PDFfiles/LottKenny.pdf

Mattos, E. (2009). Median-voter, Size the Government and Budget Spillover: Evidence for US States. Applied Economics Letter (16), 1387-1392. Recuperado de: http:// www.tandf.co.uk/journals/subscription.asp. http://econpapers.repec.org/article/ tafapeclt/v_3a16_3ay_3a2009_3ai_3a14_3ap_3a1387-1392.htm

Mccubbins, M. Kousser, T. Moule, E. (2005). Can State Tax and Expenditure Limits Effectively Reduce Spending? San Diego: Department of Political Science University of California. Recuperado de: http://spa.sagepub.com/content/8/4/331

Mccubbins, M. (1995). Putting The State Back Into State Government: The Constitution And The Budget. San Diego: University of California. Recuperado de: http://mccubbins.us/ mccubbins_files/ARTB12.PDF

Meltzer, A. Ricard, S. (1978). Why Governments Grows (and Grows) in a Democracy, National Affairs, (52), 111-118. Recuperado de: http://www.nationalaffairs.com/ public_interest/detail/why-government-grows-and-grows-in-a-democracy

Meltzer, A. Ricard, S. (1981). A Rational Theory of the Size of Government. Fournal of Political Economy. 89(5), 914-927. The University of Chicago Press. Recuperado de: http://www.jstor.org/stable/1830813

Meltzer, A. Ricard, S. (1983). Tests of a Rational Theory of the Size of Government. Public Choice, 41(3), 403-418. Recuperado de: http://repository.cmu.edu/tepper/757/ 
ISSN: 0124-3551 / Año 15, No 22 / enero-junio / pp. 87-107

Micklethwait, J. (2011). A Special Report on the Future of the State. Taming Leviathan. The Economist, 3-6. Recuperado de: http://www.economist.com/node/18359896

Moe, T. (1980). The Organization of Interests: Incentives and the Internal Dynamics of Political Interest Groups. Chicago: University of Chicago Press. http://www.amazon.com/ The-Organization-Interests-Incentives-Political/dp/0226533530

Mueller, D. Murrell, P. (1986). Interest Groups and the Size of Government. Public Choice, 48(2), 125-145. Springer. Recuperado de: http://www.jstor.org/stable/30024587

Mueller, D. (2003). Public Choice III. Cambridge: Cambridge University Press. http:// catdir.loc.gov/catdir/samples/cam031/2002022287.pdf

Nelson, M. (1986). An Empirical Analysis of State and Local Tax Structure in the Context of the Leviathan Model of Government. Public Choice, (49), 283-294. Springer. Recuperado de: http://www.jstor.org/stable/30024642

Niskanen, W. (1971). Bureaucracy and Representative Government. Chicago: Aldine-Atherton. http://books.google.com.co/books/about/Bureaucracy_and_representative_governmen.html?id=X38FAAAAMAAJ\&redir_esc $=\mathrm{y}$

Niskanen, W. (2001). Bureaucracy. In The Elgar Companion To Public Choice. William Shughart and Laura Razzolini (Eds). Northampton: Edward Elgar. Recuperado de: http://www.elgaronline.com/view/1852785403.00023.xml

Oates, W. (1972). Fiscal Federalism. New York: Harcourt Brace Jovanovich. 188191. Recuperado de: http://www.sciencedirect.com/science/article/B6V76 -458WP44-5C/2/e7416918c36dd7313f093c634la92788

Oates, W. (1989). Searching for Leviathan: A Reply and Some Further Reflections. American Economics Review, (79), 578-83. American Association. Recuperado de: http://www.jstor.org/stable/1806867

Olson, M. Jr. (1965). The logic of Collective Action: Public Goods and the Theory of Groups. Cambrige: Harvard University Press. http://books.google.es/books/about/The_logic_of_collective_action.html?id=jzTeOLtf7_wC

Pérez, M. (2002). Separación de poderes. Costos, beneficios y orden constitucional. En Debates nacionales. Álvaro Tirado Mejía (Ed.). Bogotá: Fedesarrollo - Alfaomega, pp. $1-14$.

Pérez, M. (2002). Conmemoración y evaluación de los diez años de la vigencia de la Constitución Política de Colombia. Ministerio del Interior. Dirección General de Asuntos Políticos y Electorales. Segunda Parte. 
Pérez, P. (2008). Descentralización y tamaño del sector público en España. Universidad de Cantabria. Instituto de Estudios Fiscales. Recuperado de: http://www.ief.es/documentos/recursos/publicaciones/papeles_trabajo/2008_21.pdf

Persson, T. Tabellini, G. (2005). The Economics Effects of Constitutions. Massachusetts: The MIT Press. http://www.amazon.com/Economic-Effects-Constitutions-text-TPersson-G-Tabellini/dp/B003QTFDUE

Persson, T. Tabellini, G. (2004). Constitutional Rules and Fiscal Policy Outcomes. The American Economic Review 94(1), 25-45. American Association. Recuperado de: http:// www.aeaweb.org/aer/data/march2004_tabellini_data.zip

Persson, T. \& Tabellini, G. (2002).Political Economics. Explaining Economic Policy. MIT Press. http://www.amazon.com/Political-Economics-Explaining-EconomicTabellini/dp/B00BTM9Q7G

Shelton, G. (2007). The Size and Composition of Government Expenditure. Journal of Public Expenditure, 91(11-12), 2230-2260. Elsevier. Recuperado de: http://www. sciencedirect.com/science/article/B6V76-4MWGFC4-2/2/20ee0c97e8b1c2ba8a d603f6ebf8b5e7

Spiller, P. \& Sanny, L (2008). Buy, Lobby or Sue: Interest Groups' Participation in Policy Making - A Selective Survey. En New Institutional Economics. A Guidebook. Cambridge University Press, pp. 307-327.

Wagner, A. (1911). Staatin nationalökonomischer Hinsicht. Handwörterbuch der Staatswissenschaften. Jene: Lexis.

Weingast, B., Shepsle, K. Johnson, C. (1981). The Political Economy of Benefits and Costs: A Neoclassical Approach to Redistributive Politics. Fournal of Political Economy, 89, 642-64. Recuperado de: http://www.journals.uchicago.edu/JPE/ 
